\title{
Optimization of the size and yield of graphene oxide
}

\section{sheets in the exfoliation step}

Cristina Botas, Ana M. Pérez-Mas, Patricia Álvarez, Ricardo Santamaría, Marcos Granda, Clara Blanco, and Rosa Menéndez*

Instituto Nacional del Carbón, CSIC, Apartado 73, 33080 Oviedo, Spain.

ABSTRACT. In this paper we demonstrate that the yield and size of the graphene oxide sheets (GO) obtained by sonication of graphite oxide ( $\mathrm{GrO}$ ) can be optimized not only by selecting the appropriate exfoliation conditions but also as a function of the crystalline structure of the parent graphite. A larger crystal size in the parent graphite favors GrO exfoliation and yields larger sheets in shorter sonication times, independently of the oxygen content of the GrO. A maximum yield of GO is obtained in all cases, after which no further improvement is achieved and the size of the sheets decreases.

The preparation of graphene by chemical methods, such as the graphite oxide route, offers the possibility of producing it on a large scale and, at the same time, of controlling its quality, depending on: (i) the characteristics of the parent graphite [1], (ii) the oxidation method used [2], and (iii) the final reduction of graphene oxide (GO) to graphene [3]. GO and partially reduced graphene oxides are graphene derivatives that have a structure marked by defects produced during the oxidation and/or reduction processes $[4,5]$. However, the complexity of the reactions involved in the overall process, in addition to the numerous variables that may affect the bonding of the carbon atoms in the parent graphite, make it very difficult to control the yield and quality

\footnotetext{
* Corresponding author: Tel: +34 9851190 90. E-mail: rosmenen@incar.csic.es (Rosa Menéndez)
} 
of the products (sheet size and defects). Both the size of the sheets and the defects present in the graphene material will determine its final application in fields such as electronics and composites [6].

Exfoliation of the $\mathrm{GrO}$, where there is still interaction between the graphite basal planes, is necessary to obtain GOs [6]. The application of ultrasounds and the mechanical shaking of water dispersed $\mathrm{GrO}$ are the procedures most commonly used $[6,7]$ for this purpose. It has been reported [7-11] that long mechanical shaking or sonication times propitiate the break-up of the sheets. It has also been claimed that although the severity of the oxidation process facilitates exfoliation it has a negative effect on the size of the GO sheets, as a high content of oxygen functional groups leads to more defects and bonding weakness [9]. However, to our knowledge, no studies concerning the effect of the sonication process on graphite oxides from graphites with different crystalline structure have been reported.

The aim of the present paper is to determine whether the yield and size of the GO sheets can be optimized for a specific graphite oxide by selecting appropriate exfoliation conditions. We report on: (i) the use of two graphites of different crystalline structure, a natural (NG) and a synthetic graphite (SG) as raw materials; (ii) the preparation of their respective GrOs; (iii) the exfoliation of the two GrOs by subjecting them to ultrasounds for different periods of time to obtain the GOs and (iv) the quantification and characterization of the GOs produced.

The X-ray diffraction results show that $\mathrm{NG}$ is more crystalline $\left(\mathrm{L}_{\mathrm{a}}, 82.8 \mathrm{~nm}\right.$ and $\left.\mathrm{L}_{\mathrm{c}}, 59.5 \mathrm{~nm}\right)$ than SG ( $\mathrm{L}_{\mathrm{a}}, 65.4 \mathrm{~nm}$ and $\left.\mathrm{L}_{\mathrm{c}}, 26.2 \mathrm{~nm}\right)$. The GrOs prepared from both graphites by a modified Hummers method $[1,12]$ show similar $\mathrm{C} / \mathrm{O}$ ratios, as determined by XPS $(\approx 2)$. However, the oxide obtained from the natural graphite of larger crystal size ( $\mathrm{NGrO})$ has a larger amount $\mathrm{sp}^{3} \mathrm{C}$ $\mathrm{C}$ bonds $(12.8 \%$ in $\mathrm{NGrO}$ and $4.9 \%$ in $\mathrm{SGrO})$ and $\mathrm{C}-\mathrm{OH}$ bonds that are probably located in the 
interior of the basal planes of the $\mathrm{GrO}(36.2 \%$ in $\mathrm{NGrO}$ and $1.8 \%$ in SGrO). In contrast, SGrO contains a larger amount of C-O-C bonds (39.4\% in SGrO and $14.3 \%$ in NGrO) and carboxylic groups located at the edges of the sheets ( $10.7 \%$ in $\mathrm{SGrO}$ and $4.4 \%$ in $\mathrm{NGrO})$. The GrOs were dispersed in purified water and subjected to ultrasounds for the following periods of time: $0.5 \mathrm{~h}$, $1 \mathrm{~h}, 2 \mathrm{~h}, 4 \mathrm{~h}, 8 \mathrm{~h}, 10 \mathrm{~h}$ and $24 \mathrm{~h}$ (see Supporting information, S.I.). The concentration of GO sheets after each sonication period was quantitatively determined in both samples by UV-Vis to measure the exfoliation yield (see S.I.) and the sheets were imaged using an atomic force microscope (AFM, see S.I.).

It was found that, although the amount of GO sheets increases with increasing sonication time for both samples, the trend in each case is quite different (Figure 1a). NGrO exfoliates much rapidly from the very beginning and reaches a maximum yield of graphene oxide (NGO) in about $6 \mathrm{~h}(84 \%)$, but with no further improvement after that. In the case of SGrO there is a substantial increase during the first $10 \mathrm{~h}$ and then a more gradual increase for up to $24 \mathrm{~h}$. At this point it exhibits an exfoliation yield of $43 \%$ which is nearly half that of NGO.
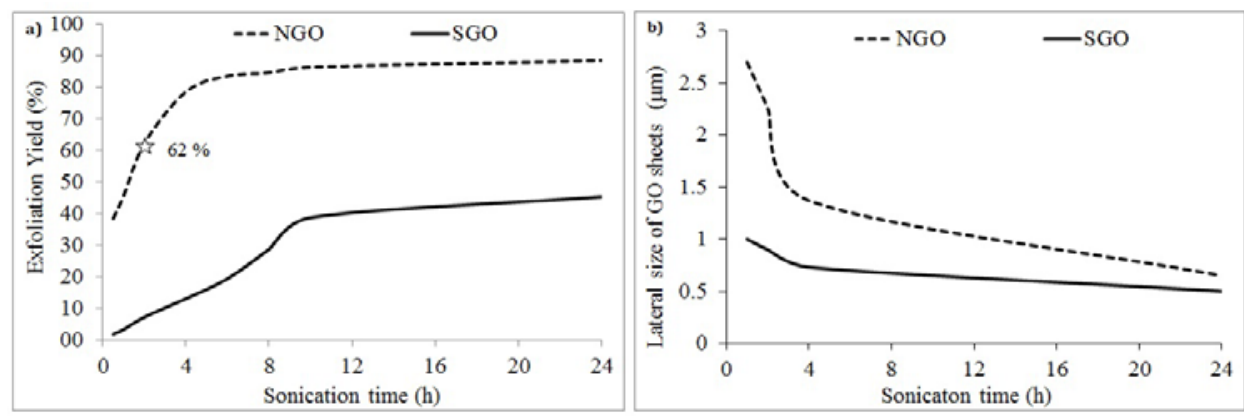

Figure 1. a) Exfoliation Yield of GO and b) lateral size of GO sheets, vs sonication time.

The lateral size of the GO sheets as determined by AFM (Figure 1b), undergoes a sharp decrease in NGO in the first $4 \mathrm{~h}$ of sonication (from $2 \mu \mathrm{m}$ to $1 \mu \mathrm{m}$ ), after which it continues to decrease but more gradually. In the case of SGO, there is also a sharp decrease in the first $4 \mathrm{~h}$ of sonication (but less pronounced), after which no further reduction in size is observed. The SGO 
sheets are considerably smaller at short sonication times (below $4 \mathrm{~h}$ ) than those of NGO. However, as sonication proceeds, the differences between the lateral size of the GOs decrease. After $6 \mathrm{~h}$, the sheets of both GOs are similar in size, indicating that the duration of the sonication treatment has a more pronounced effect on large sheets.

The AFM images clearly illustrate the yield and size of the sheets in the two GO samples (Figure 2). For short sonication times, NGO shows a higher population of large sheets (monolayers) than SGO. As the sonication time increases, the size of the sheets is similar in both samples although the size of the population is still significantly lower in the case of SGO.
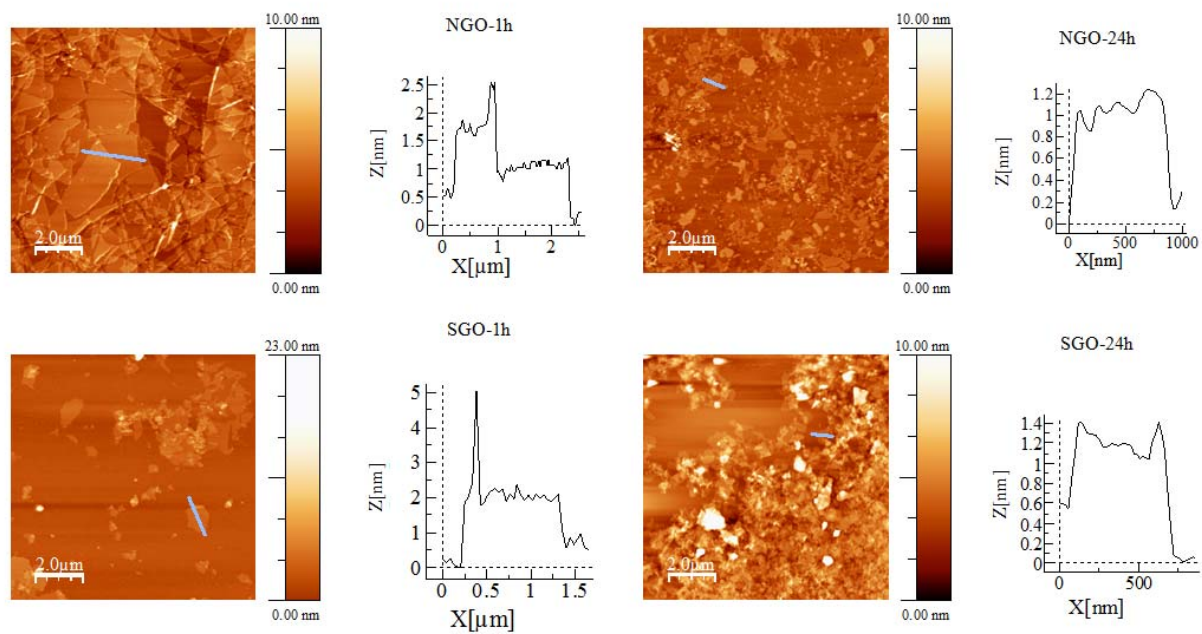

SGO-24h

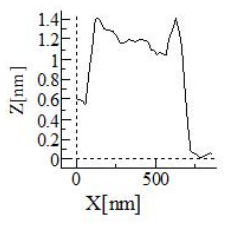

Figure 2. AFM images of the GO sheets after $1 \mathrm{~h}$ and $24 \mathrm{~h}$ of sonication and height profiles corresponding to the sections indicated by the blue lines.

Our findings suggest that each $\mathrm{GrO}$ requires a specific sonication time to reach its maximum yield of GO. If this limit is exceeded, instead of improving the exfoliation yield, it has a deleterious effect on the size of the sheets. Moreover, the larger crystal size in the parent graphite favours GrO exfoliation and yields larger GO sheets with shorter sonication times, regardless of the $\mathrm{C} / \mathrm{O}$ ratio of the GrO. The better behavior of $\mathrm{NGrO}$ can be attributed to the larger amount of hydroxyl groups (36.2\% versus $1.8 \%$ in SGrO), preferentially located in the interior of the basal 
plane, with a longer bonding distance than epoxy groups (more abundant in SGrO), which facilitates the exfoliation process. In addition, the larger amount of oxygen functional groups (hydroxyl and epoxy) in the basal plane creates areas of weakness that contribute to the break-up of the sheets when sonicated for longer times [9] (see mechanism proposed in S.I.). These results provide a guidance for the selection of the parent graphite and the exfoliation conditions depending on the size of the graphene sheets required for a specific application (i.e. microelectronics or catalysis).

ACKNOWLEDGMENT: The authors thank MICINN (CONSOLIDER INGENIO 2010, Ref. CSD2009-00050, MAT2010-16194) for their financial support. Dr. Patricia Alvarez thanks MICINN for her Ramon y Cajal contract.

\section{REFERENCES}

[1] Botas C, Álvarez P, Blanco C, Santamaría R, Granda M, Ares P, et al. The effect of the parent graphite on the structure of graphene oxide. Carbon 2012; 50: 275-82.

[2] Dreyer DR, Park S, Bielawski CW, Ruoff RS. The chemistry of graphene oxide. Chem. Soc Rev 2010; 39: 228-40.

[3] Pei S, Cheng H. The reduction of graphene oxide. Carbon 2012; 50: 3210-3228.

[4] Mao S, Pu H, Chen J. Graphene oxide and its reduction: modeling and experimental progress. RSC Adv 2012; 2: 2643-62.

[5] Botas C, Álvarez P, Blanco C, Gutiérrez MD, Ares P, Zamani R, et al. Tailored graphene materials by chemical reduction of graphene oxides of different atomic structure. RSC Adv 2012; 2:9643-50.

[6] Inagaki M, Kim YA, Endo M. Graphene: preparation and structural perfection. J Mater Chem 2011; 21: 3280-94.

[7] Cai M, Thorpe D, Adamson DH, Schniepp HC. Methods of graphite exfoliation. J Mater Chem 2012; 22: 24992-5002.

[8] Zhao JPS, Ren W, Gao L. Chen, H. Efficient Preparation of Large-Area Graphene Oxide Sheets for Transparent Conductive Films. ACS Nano 2010; 4: 5245-52.

[9] Pan S, Aksay IA. Factors Controlling the Size of Graphene Oxide Sheets Produced via the Graphite Oxide Route. ACS Nano 2011; 5(5): 4073-83.

[10] Qi GQ, Cao J, Bao RY, Liu ZY, Yang W, Xie BH, Yang MB. Tuning the structure of graphene oxide and the properties of poly(vinyl alcohol)/graphene oxide nanocomposites by ultrasonication. J Mater Chem A 2013; 1: 3163-70.

[11] McAllister, MJ, Li JL, Adamson DH, Schniepp HC, Abdala AA, Liu J, Herrera-Alonso M, Milius D L, Car R, Prud'homme RK, Aksay IA. Single Sheet Functionalized Graphene by Oxidation and Thermal Expansion of Graphite. Chem Mater 2007; 19: 4396-404. 
[12] Hummers WS, Offeman RE. Preparation of Graphitic Oxide. J Am Chem Soc 1958; 80: $1339-40$. 